九州大学学術情報リポジトリ

Kyushu University Institutional Repository

\title{
Freezing Tolerance of Transgenic Tobacco with Increased Content of Unsaturated Fatty Acid by Expressiong the CvFad3 Gene
}

Shimizu, Hideyuki

Laboratory of Food Hygienic Chemistry, Division of Food Biotechnology, Department of

Bioscience and Biotechnology, Faculty of Agriculture, Graduate School, Kyushu University

Furuya, Naoki

Suga, Koushirou

Laboratory of Food Hygienic Chemistry, Division of Food Biotechnology, Department of

Bioscience and Biotechnology, Faculty of Agriculture, Graduate School, Kyushu University

Honjoh, Ken-ichi

他

https://doi.org/10.5109/4500

出版情報：九州大学大学院農学研究院紀要. 47 (2)，pp.307-317，2003-02-01. Faculty of Agriculture, Kyushu University

バージョン：

権利関係 : 


\title{
Freezing Tolerance of Transgenic Tobacco with Increased Content of Unsaturated Fatty Acid by Expressing the CvFad2 or CvFad3 Gene
}

\author{
Hideyuki SHIMIZU*, Naoki FURUYA*, Koushirou SUGA***, \\ Ken-ichi HONJOH ${ }^{\dagger}$, Takahisa MIYAMOTO, \\ Shoji HATANO*** and Masayoshi IIO
}

\author{
Laboratory of Food Hygienic Chemistry, Division of Food Biotechnology, Department of \\ Bioscience and Biotechnology, Faculty of Agriculture, Graduate School, \\ Kyushu University, Fukuoka 812-8581, Japan \\ (Received October 31, 2002 and accepted November 7, 2002)
}

\begin{abstract}
Two fatty acid desaturases were independently expressed in tobacco plants and freezing tolerance of the transgenic plants was investigated: the two desaturases were $\mathrm{CvFAD2}$, which desaturates microsomal C18:1 (oleic acid) to C18:2 (linoleic acid), and CvFAD3, which desaturates microsomal C18:2 to C18:3 (linolenic acid). Fatty acid composition analysis showed that the $\mathrm{C} 18: 2$ contents in the plants transformed with $\mathrm{pBE} 2113 / \mathrm{CvFad} 2$ did not increase compared with that of the wild-type plant. However, an increase in the C18:3 content by $2.4 \%$ was observed in the line of No. 19 although the increase was not statistically significant. In the plant transformed with pBE2113/CvFad3, the C18:3 content increased by $6.4 \%$ compared with that of the wild-type plant. Measurement of the electrolyte leakage of the leaves showed that freezing tolerance of the CvFad2 plant was a little higher than that of the wild-type plant at all temperatures investigated (from -1 to $-4^{\circ} \mathrm{C}$ ). Contrary to the case of the $C v F a d 2$, the freezing tolerance of the $\mathrm{CvFad} 3$ plant tended to be slightly lower than that of the wild-type at all temperatures tested despite the unsaturation levels of the plant were higher than those of the CvFad2 plants.
\end{abstract}

\section{ABBREVIATIONS}

FAD, fatty acid desaturase; G6PDH, glucose 6-phosphate dehydrogenase; GPx, glutathione peroxidase; $\mathrm{PC}$, phosphatidylcholine; $\mathrm{SOD}$, superoxide dismutase

\section{INTRODUCTION}

Freezing stress is one of the most severe environmental stresses and cold-sensitive plants suffer serious damage by freezing. But some plant species develop freezing tolerance by cold acclimation when they are exposed to non-freezing low temperatures and survive freezing (Wanner and Junttila, 1999, Webb et al., 1994). During the acclimation, expressions of many genes are induced and various biochemical and physiological changes occur (Jaglo-Ottosen et al., 1998, Seki et al., 2001, Guy, 1990). Among these

\footnotetext{
* Laboratory of Food Hygienic Chemistry, Division of Food Biotechnology, Department of Bioscience and Biotechnology, Graduate School of Bioresource and Bioenvironmental Sciences, Kyushu University, Fukuoka 812-8581, Japan

** Chlorella Industries Co. Ltd., 1343 Hisatomi, Chikugo, Fukuoka 833-0056, Japan

*** Graduate School of Health and Social Welfare Science, Nishikyushu University, 4490-9 Ooazaozaki, Kanzaki-machi, Kanzaki-gun, Saga 842-8585, Japan

+ Corresponding author (E-mail: honjoh@agr.kyushu-u.ac.jp)
} 
changes, increase in the level of polyunsaturated fatty acids such as C18:3 is a major change and observed in many plants (Graham and Patterson, 1982).

We have studied the development of freezing tolerance in plants using Chlorella vulgaris C-27, which is useful as an eukaryotic model. Hardened cells of C. vulgaris C-27 can survive slow freezing to $-196^{\circ} \mathrm{C}$ (Hatano et al., 1976). During this hardening period, several changes including up-regulation of $\mathrm{CvFad2}$ and $\mathrm{CvFad} 3$ genes (Suga et al., 2002), accumulation of HIC6 protein (Honjoh et al., 1995) and an increase in G6PDH activity (Sadakane et al., 1980) occur. CvFAD2 and CvFAD3 desaturate microsomal C18:1 to C18:2 and microsomal C18:2 to C18:3, respectively.

The increase in unsaturation level of fatty acids is thought to be important for development of cold tolerance in plants. The fad2 mutant of Arabidopsis, which is deficient in the activity of the microsomal C18:1 desaturase, failed to undergo stem elongation at $12{ }^{\circ} \mathrm{C}$ and gradually died at $6{ }^{\circ} \mathrm{C}$ although it showed very similar growth to a wild-type (Miquel et al., 1993). On the other hand, $F A D 7$ transgenic tobacco, having increased contents of $\mathrm{C} 16: 3$ and C18:3 in plastid membrane lipids, showed decreased leaf chlorosis induced by low temperature treatment (Kodama et al., 1995). As well as increasing cold tolerance, fatty acids desaturation seems to be important for development of freezing tolerance. Freezing tolerance of protoplasts isolated from nonacclimated rye leaves was increased by an artificial enrichment of the plasma membrane with 18:2/18:2-PC or 18:3/18:3-PC (Steponkus et al., 1988). However, definite evidence that fatty acids desaturation increases freezing tolerance in plants has not been shown until now.

In this study, in order to make clear the contribution of increase of unsaturated fatty acids in plasma membrane to freezing tolerance in plants, we tried to make tobacco plants with high content of unsaturated fatty acids by introduction of the CvFad2 or CvFad3 gene.

\section{MATERIALS AND METHODS}

\section{Construction of pBE2113/CvFad2 plasmid}

The CvFad2 was mutated, using PCR, to introduce a BamHI site just before the ATG start codon and a $S a c I$ site after the stop codon. For this purpose, two oligonucleotides, 5'-AGCAGGGGATCCATGGCTGCAACCCGG-3' and 5'-CCGGAGAGCTCACGCTATACTCCCAGCACC-3', were synthesized as primers. The open reading frame of the CvFad2 was amplified by PCR, using KOD Dash DNA polymerase (Toyobo, Tokyo, Japan), then the amplified fragment was subcloned into a pGEM-T Easy Vector (Promega, Madison, WI, USA). After confirming the sequence of the pGEM-T Easy Vector/CvFad2, the plasmid was digested with a combination of $B a m \mathrm{HI}$ and $S a c \mathrm{I}$, and the BamHI-SacI fragment containing CvFad2 was ligated into the BamHI-SacI-digested pBE2113 plasmid vector (Mitsuhara et al., 1996). Escherichia coli HB101 was used for the propagation of this plasmid.

pBE2113/CvFad3 was constructed as described previously. (Suga et al., 2002)

\section{Plant transformation and growth conditions}

The pBE2113/CvFad2 and pBE2113/CvFad3 transgenic tobacco plants (Nicotiana 
tabacum var. Samsun) were constructed via Agrobacterium tumefaciens LBA4404, as described previously (Honjoh et al., 2001). The wild-type tobacco plants were cultured on MS agar medium and the transgenic were on MS agar medium supplemented with $100 \mu \mathrm{g} / \mathrm{ml}$ kanamycin and $250 \mu \mathrm{g} / \mathrm{ml}$ carbenicillin, under the conditions described previously (Honjoh et al., 2001). For freezing tolerance tests, the tobacco plants were grown in pots filled with a mixture of perlite and vermiculite $(1: 1, \mathrm{v} / \mathrm{v})$.

\section{Southern hybridization analysis of $\mathrm{CvFad} 2$ and $\mathrm{CvFad} 3$ genes}

Southern hybridization analysis was done as described previously (Honjoh et al., 2001). Thirty five $\mu \mathrm{g}$ of genomic DNA were digested with HindIII (for CvFad2) or EcoRI (for $\mathrm{CvFad3}$ ), then separated on a 1.0\% agarose gel. The separated DNA was transferred onto a Hybond-N+ nylon membrane (Amersham, Buckinghamshire, England) and hybridized with the ${ }^{32} \mathrm{P}$-labeled CvFad2 or CvFad3 probe.

\section{Northern hybridization analysis of $\mathrm{CvFad} 2$ and $\mathrm{CvFad} 3$ genes}

Total RNAs were prepared from leaves by using "Sepasol RNA I Super" (Nacalai tesque, Kyoto, Japan), according to the manufacturer's instruction. Forty $\mu$ g of the RNAs were separated on a $1.2 \%$ agarose gel containing formaldehyde, transferred to a Hybond-N+nylon membrane, and hybridized with the ${ }^{32} \mathrm{P}$-labeled CvFad2 or CvFad3 probe.

\section{Fatty acid composition analysis of leaf tissue}

For lipids extraction, healthy green leaves were placed in a $2 \mathrm{ml}$ microtube and heated in a boiling bath for $3 \mathrm{~min}$. Then the tube was immersed in liquid nitrogen and the leaves were ground. One point eight $\mathrm{ml}$ of chloroform/methanol $(2: 1, \mathrm{v} / \mathrm{v})$ was added, and the sample was incubated at $40^{\circ} \mathrm{C}$ for $30 \mathrm{~min}$ in the dark and centrifuged at $13,800 \times \mathrm{g}$ for $20 \mathrm{~min}$ at $4^{\circ} \mathrm{C}$. The supernatant was divided equally and transferred to two new microtubes, to each of which $220 \mu \mathrm{l}$ of water was added. After centrifugation at $1,500 \times g$ for $10 \mathrm{~min}$ at $4^{\circ} \mathrm{C}$, each of the lower chloroform phases was transferred to a new tube, vacuum dried and redissolved in $100 \mu \mathrm{l}$ of chloroform. The two solutions were combined and transferred to a screwcapped centrifuge tube. For lipids methanolysis, the sample was mixed with $400 \mu \mathrm{l}$ of $5 \%(\mathrm{v} / \mathrm{v}) \mathrm{HCl}$ in methanol. The sample was boiled for $2 \mathrm{~h}$, then mixed with $1 \mathrm{ml}$ of $0.9 \%(\mathrm{w} / \mathrm{v}) \mathrm{NaCl}$ solution and $400 \mu \mathrm{l}$ of hexane were added and centrifuged at $1,500 \times g$ for $10 \mathrm{~min}$. The upper organic phase was transferred to a new microtube and the lower aqueous phase was re-extracted with $100 \mu \mathrm{l}$ of hexane. The two extracts were combined, condensed by drying under $\mathrm{N}_{2}$ down to $250 \mu \mathrm{l}$, and then analyzed with a gas chromatograph (model GC-9A; Shimadzu, Kyoto, Japan), as described in Joh et al. (1993).

\section{Measurements of the freezing tolerance of the leaves}

Freezing tolerance of the tobacco leaves was assessed by measuring the electrolyte leakage (EL), as described previously (Honjoh et al., 2001). 


\section{RESULTS}

\section{Production of transformed tobacco with pBE2113/CvFad2}

In order to obtain plants with high contents of polyunsaturated fatty acids, a gene coding for CvFAD2 (Suga et al., 2002), which desaturates C18:1 to C18:2, was introduced into tobacco plants. In this process, a pBE2113 vector (Mitsuhara et al., 1996), which has a highly efficient promoter, was used. Through the transformation with pBE2113/CvFad2, eight independent lines of plant were regenerated.

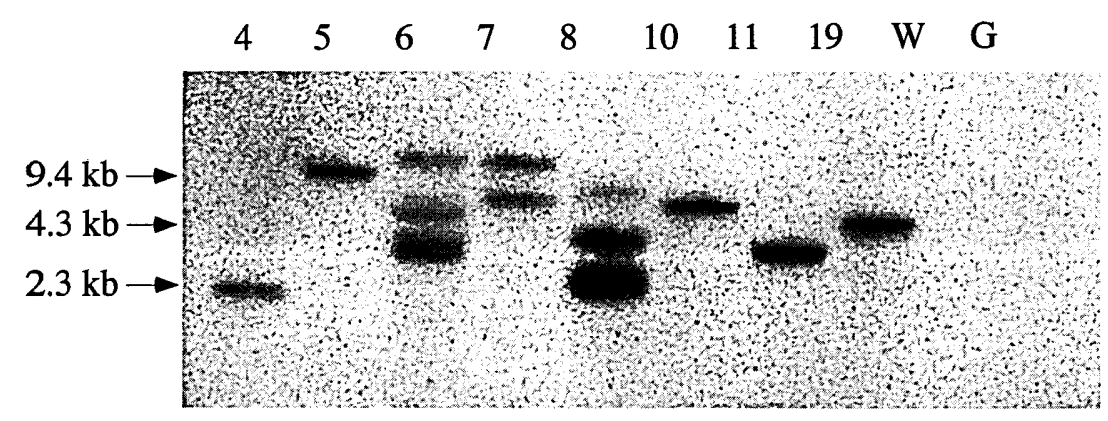

Fig. 1. Genomic Southern blot analysis to show the integration of the CvFad2 gene into the genome of the tobacco plants transformed with pBE2113/CvFad2. Genomic DNA (35 $\mu \mathrm{g})$ was digested with HindIII and detected with a ${ }^{32} \mathrm{P}$-labeled CvFad2 probe. The identification numbers of the transgenic lines are indicated on the top. W, wild-type tobacco; G, pBE2113-GUS transformed tobacco.

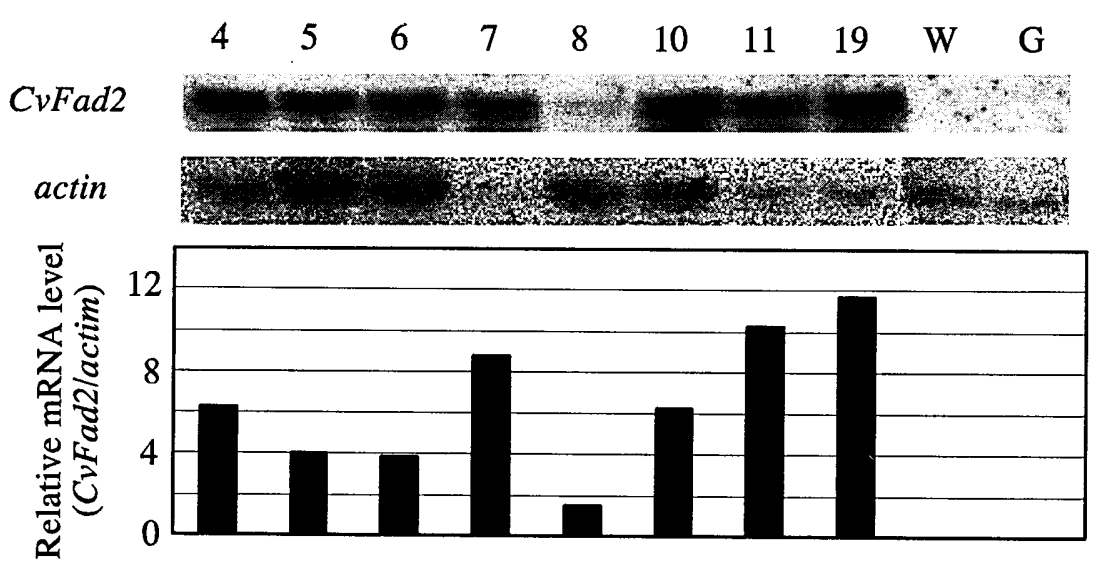

Fig. 2. Northern blot analysis of CvFad2 in tobacco plants transformed with pBE2113/CvFad2. Total RNA (40 $\mu$ g) was subjected to gel blot analysis using a ${ }^{32} \mathrm{P}-$ labeled $C v F a d 2$ probe. Then blots were stripped and reprobed with the fragment of actin gene as a loading control. The identification numbers of the transgenic lines are indicated on the top. W, wild-type tobacco; G, pBE2113-GUS transformed tobacco. 
To confirm the incorporation of the CvFad2 gene into the genomic DNA of the transgenic tobacco plants, the plants were analyzed by genomic Southern hybridization analysis using a ${ }^{32} \mathrm{P}$-labeled $C v F a d 2$ probe (Fig. 1). All transgenic plants showed at least one hybridization band; one hybridization band was observed in five clones (Nos. 4, 5, 10 , 11 and 19), two bands in one clone (No. 7) and three bands in two clones (Nos. 6 and 8).

To make sure the expression of $C v F a d 2$, total RNAs were prepared from tobacco plants and northern hybridization analysis was done (Fig. 2). In all CvFad2 transgenic plants, only a single band was detected with the ${ }^{32} \mathrm{P}-$ labeled CvFad2 probe. Especially, No. 19 line showed the highest expression level although this line had only one copy of the CvFad2 gene. In contrast, lines of No. 6 and 8, each of which had three Southern hybridization bands, showed relatively low expression levels. These results showd that CvFad2 was integrated into the genomic DNA and expressed in the transgenic tobacco plants.

To investigate the fatty acid composition of these plants, fatty acid methyl esters were prepared from the leaf tissues and analyzed by gas chromatography. The wild-type and pBE2113-GUS transgenic plants were used as the control plants. The result of the fatty acid composition analysis is shown in Table 1. In all CvFad2 plants, there was no increase in the C18:2 content. But, in two clones (Nos. 5 and 19), the C18:1 content decreased and an increase in the C18:3 content was observed although any of the changes is not significant. So it was supposed that C18:2, which had been desaturated from C18:1 by CvFAD2, was desaturated into C18:3 by endogenous FAD3.

Table 1. Fatty acid composition of leaf tissues from CvFad2 transgenic tobacco plants

\begin{tabular}{|c|c|c|c|c|c|c|c|c|}
\hline \multirow{2}{*}{ Clone } & \multicolumn{7}{|c|}{ Fatty acid composition (\%) } & \multirow[b]{2}{*}{$18: 3$} \\
\hline & $16: 0$ & $16: 1$ & $16: 2$ & $16: 3$ & $18: 0$ & $18: 1$ & $18: 2$ & \\
\hline Wild-type & $14.3 \pm 0.6$ & $1.2 \pm 0.1$ & $1.9 \pm 0.2$ & $7.1 \pm 0.6$ & $2.3 \pm 0.4$ & $1.6 \pm 0.2$ & $15.0 \pm 1.5$ & $56.7 \pm 1.5$ \\
\hline pBE2113-GUS & $18.5 \pm 2.4$ & $0.6 \pm 0.1$ & $0.8 \pm 0.2$ & $3.9 \pm 0.6$ & $2.9 \pm 0.2$ & $1.7 \pm 0.3$ & $16.9 \pm 0.1$ & $54.9 \pm 3.5$ \\
\hline pBE2113/CvFad2 No. 4 & $14.3 \pm 0.3$ & $1.8 \pm 0.0$ & $1.5 \pm 0.0$ & $8.5 \pm 0.2$ & $1.5 \pm 0.0$ & $1.4 \pm 0.0$ & $14.5 \pm 0.2$ & $55.9 \pm 0.1$ \\
\hline pBE2113/CvFad2 No. 5 & $15.1 \pm 0.8$ & $1.4 \pm 0.2$ & $1.2 \pm 0.2$ & $5.6 \pm 0.3$ & $1.5 \pm 0.2$ & $1.1 \pm 0.1$ & $14.8 \pm 1.4$ & $59.0 \pm 3.3$ \\
\hline pBE2113/CvFad2 No. 6 & $14.7 \pm 0.4$ & $2.2 \pm 0.0$ & $1.6 \pm 0.1$ & $8.5 \pm 0.3$ & $1.4 \pm 0.1$ & $1.0 \pm 0.1$ & $13.7 \pm 0.2$ & $56.8 \pm 0.5$ \\
\hline pBE2113/CvFad2 No. 7 & $15.2 \pm 0.1$ & $1.5 \pm 0.0$ & $1.2 \pm 0.0$ & $5.9 \pm 0.1$ & $1.6 \pm 0.0$ & $1.3 \pm 0.0$ & $15.0 \pm 0.1$ & $58.0 \pm 0.6$ \\
\hline pBE2113/CvFad2 No. 8 & $15.5 \pm 0.5$ & $1.5 \pm 0.1$ & $1.6 \pm 0.1$ & $7.0 \pm 0.2$ & $2.5 \pm 0.3$ & $2.3 \pm 0.3$ & $13.6 \pm 1.7$ & $55.9 \pm 3.1$ \\
\hline pBE2113/CvFad2 No. 10 & $16.2 \pm 0.8$ & $0.8 \pm 0.1$ & $1.5 \pm 0.2$ & $6.0 \pm 0.1$ & $2.8 \pm 0.2$ & $2.8 \pm 0.1$ & $16.7 \pm 0.2$ & $53.1 \pm 0.4$ \\
\hline pBE2113/CvFad2 No. 11 & $17.0 \pm 0.5$ & $1.1 \pm 0.2$ & $1.2 \pm 0.1$ & $5.3 \pm 0.1$ & $1.7 \pm 0.2$ & $1.4 \pm 0.1$ & $16.4 \pm 0.2$ & $55.8 \pm 0.2$ \\
\hline pBE2113/CvFad2 No. 19 & $14.3 \pm 0.7$ & $1.6 \pm 0.1$ & $1.8 \pm 0.0$ & $7.7 \pm 0.6$ & $2.3 \pm 0.0$ & $0.9 \pm 0.0$ & $12.3 \pm 1.0$ & $59.1 \pm 1.1$ \\
\hline
\end{tabular}

The values are $\operatorname{mol} \% \pm \mathrm{SD}(\mathrm{n}=3)$

\section{Production of transformed tobacco with pBE2113/CvFad3}

Introduction of a gene coding for CvFAD3 (Suga et al., 2002), which desaturates C18:2 to C18:3, into tobacco plants was also done in the same way as CvFad2 introduction. By the transformation with pBE2113/CvFad3, ten independent lines of plants were obtained.

Incorporation of the CvFad3 gene into the genomic DNA of the tobacco plants was 
confirmed by genomic Southern hybridization analysis using a ${ }^{32} \mathrm{P}$-labeled CvFad3 probe (Fig. 3). Unlike the case of the CvFad2 plants, a hybridization band of $2.7 \mathrm{~kb}$ was detected commonly in all plants including the wild-type and pBE2113-GUS plants. This band was probably due to an endogenous fad3 gene which was detected by cross-hybridization with the probe. Except the common band, other bands were observed only in the CvFad3 transgenic plants; one hybridization band was observed in six clones (Nos. 4, 5, $9,11,14$ and 15), two bands in three clones (Nos. 7, 13 and 17) and three in one clone (No. 12).

Then, expression of $\mathrm{CvFad} 3$ was investigated by northern hybridization analysis (Fig.

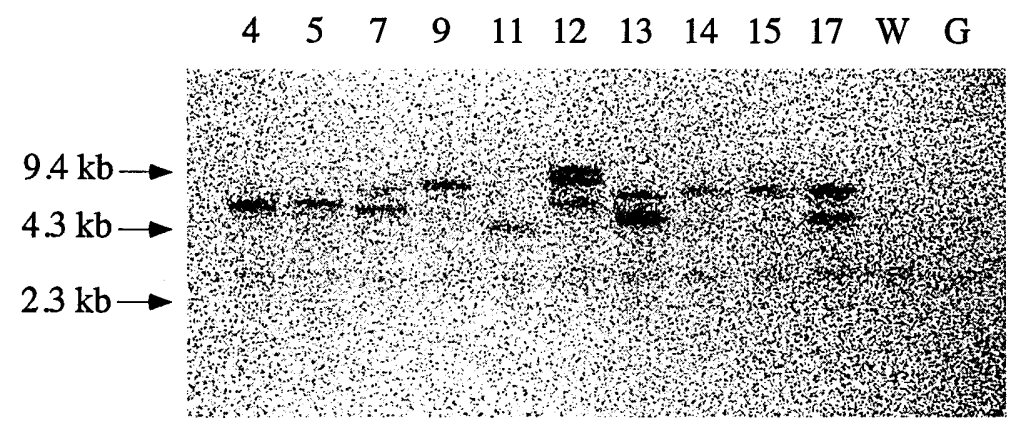

Fig. 3. Genomic Southern blot analysis to show the integration of the CvFad3 gene into the genome of the tobacco plants transformed with pBE2113/CvFad3. Genomic DNA $(35 \mu \mathrm{g})$ was digested with EcoRI and detected with a ${ }^{32} \mathrm{P}$-labeled CvFad3 probe. For other details, see Fig. 1.

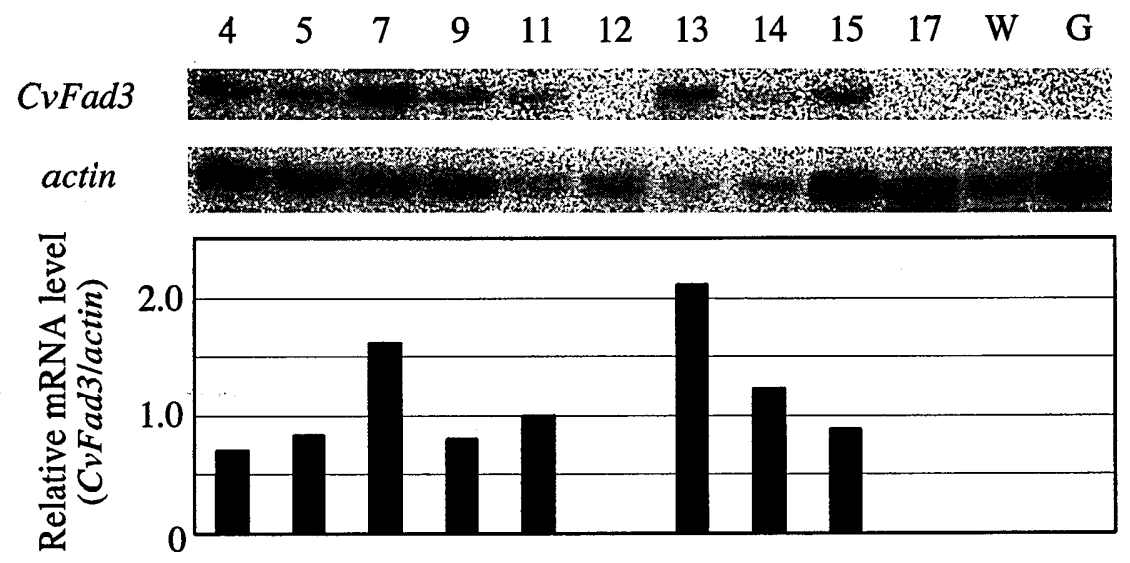

Fig. 4. Northern blot analysis of CvFad3 in tobacco plants transformed with pBE2113/CvFad3. Total RNA $(40 \mu \mathrm{g})$ was subjected to gel blot analysis using a ${ }^{32} \mathrm{P}-$ labeled $C v F a d 3$ probe. For other details, see Fig. 2. 
4). Eight clones (Nos. 4, 5, 7, 9, 11, 13, 14 and 15) showed single hybridization bands. But two clones (Nos. 12 and 17) revealed no hybridization band in spite of CvFad3 incorporation into the clones.

As shown in Table 2, an increase in the level of C18:3 was observed in four plants (Nos. 4, 7, 9 and 15). In No. 7 line, which showed the highest content of C18:3 (63.1\%), the $\mathrm{C} 18: 3$ content increased by $6.4 \%$ compared with the wild-type. In connection with the increase, the level of $\mathrm{C} 18: 2$ decreased by $5.2 \%$.

Table 2. Fatty acid composition of leaf tissues from CvFad3 transgenic tobacco plants

\begin{tabular}{lcccccccc}
\hline \multirow{2}{*}{ Clone } & \multicolumn{7}{c}{ Fatty acid composition (\%) } \\
\cline { 2 - 8 } & $16: 0$ & $16: 1$ & $16: 2$ & $16: 3$ & $18: 0$ & $18: 1$ & $18: 2$ & $18: 3$ \\
\hline Wild-type & $14.3 \pm 0.6$ & $1.2 \pm 0.1$ & $1.9 \pm 0.2$ & $7.1 \pm 0.6$ & $2.3 \pm 0.4$ & $1.6 \pm 0.2$ & $15.0 \pm 1.5$ & $56.7 \pm 1.5$ \\
pBE2113-GUS & $18.5 \pm 2.4$ & $0.6 \pm 0.1$ & $0.8 \pm 0.2$ & $3.9 \pm 0.6$ & $2.9 \pm 0.2$ & $1.7 \pm 0.3$ & $16.9 \pm 0.1$ & $54.9 \pm 3.5$ \\
pBE2113/CvFad3 No. 4 & $15.5 \pm 1.5$ & $1.7 \pm 0.5$ & $1.1 \pm 0.2$ & $5.2 \pm 0.8$ & $2.8 \pm 0.8$ & $1.6 \pm 0.4$ & $11.4 \pm 3.7$ & $60.7 \pm 4.1$ \\
pBE2113/CvFad3 No. 5 & $15.9 \pm 0.8$ & $2.2 \pm 0.0$ & $1.4 \pm 0.0$ & $6.6 \pm 0.5$ & $1.9 \pm 0.1$ & $1.2 \pm 0.3$ & $14.4 \pm 0.8$ & $56.3 \pm 2.0$ \\
pBE2113/CvFad3 No. 7 & $14.6 \pm 2.0$ & $1.9 \pm 0.2$ & $1.1 \pm 0.1$ & $6.4 \pm 1.1$ & $2.0 \pm 0.5$ & $1.0 \pm 0.7$ & $9.8 \pm 0.6$ & $63.1 \pm 1.4$ \\
pBE2113/CvFad3 No.9 & $16.3 \pm 0.3$ & $2.1 \pm 0.1$ & $1.0 \pm 0.2$ & $7.1 \pm 0.7$ & $2.2 \pm 0.2$ & $0.7 \pm 0.3$ & $10.2 \pm 1.7$ & $60.3 \pm 1.6$ \\
pBE2113/CvFad3 No. 11 & $14.4 \pm 3.1$ & $1.9 \pm 0.1$ & $1.6 \pm 0.3$ & $7.0 \pm 0.2$ & $2.0 \pm 0.3$ & $1.1 \pm 0.4$ & $13.1 \pm 0.2$ & $58.7 \pm 2.3$ \\
pBE2113/CvFad3 No. 12 & $16.4 \pm 1.1$ & $1.8 \pm 0.2$ & $1.3 \pm 0.1$ & $5.8 \pm 0.5$ & $2.6 \pm 0.6$ & $0.8 \pm 0.1$ & $13.1 \pm 0.4$ & $58.3 \pm 0.8$ \\
pBE2113/CvFad3 No. 13 & $14.7 \pm 2.3$ & $1.6 \pm 0.3$ & $1.3 \pm 0.2$ & $6.6 \pm 0.9$ & $2.5 \pm 0.3$ & $1.7 \pm 0.6$ & $14.6 \pm 1.8$ & $57.0 \pm 2.7$ \\
pBE2113/CvFad3 No. 14 & $16.9 \pm 1.4$ & $1.5 \pm 0.2$ & $1.2 \pm 0.0$ & $5.1 \pm 0.6$ & $4.1 \pm 1.6$ & $2.0 \pm 0.9$ & $11.3 \pm 1.9$ & $57.8 \pm 2.2$ \\
pBE2113/CvFad3 No. 15 & $14.0 \pm 1.0$ & $1.6 \pm 0.4$ & $1.3 \pm 0.0$ & $6.0 \pm 0.5$ & $1.8 \pm 0.1$ & $1.0 \pm 0.1$ & $13.1 \pm 2.0$ & $61.0 \pm 2.7$ \\
pBE2113/CvFad3 No. 17 & $18.0 \pm 0.8$ & $1.9 \pm 0.4$ & $1.9 \pm 0.6$ & $4.7 \pm 1.4$ & $2.7 \pm 1.4$ & $1.0 \pm 0.1$ & $12.5 \pm 0.9$ & $57.3 \pm 1.5$ \\
\hline
\end{tabular}

The values are $\mathrm{mol} \% \pm \mathrm{SD}(\mathrm{n}=3)$

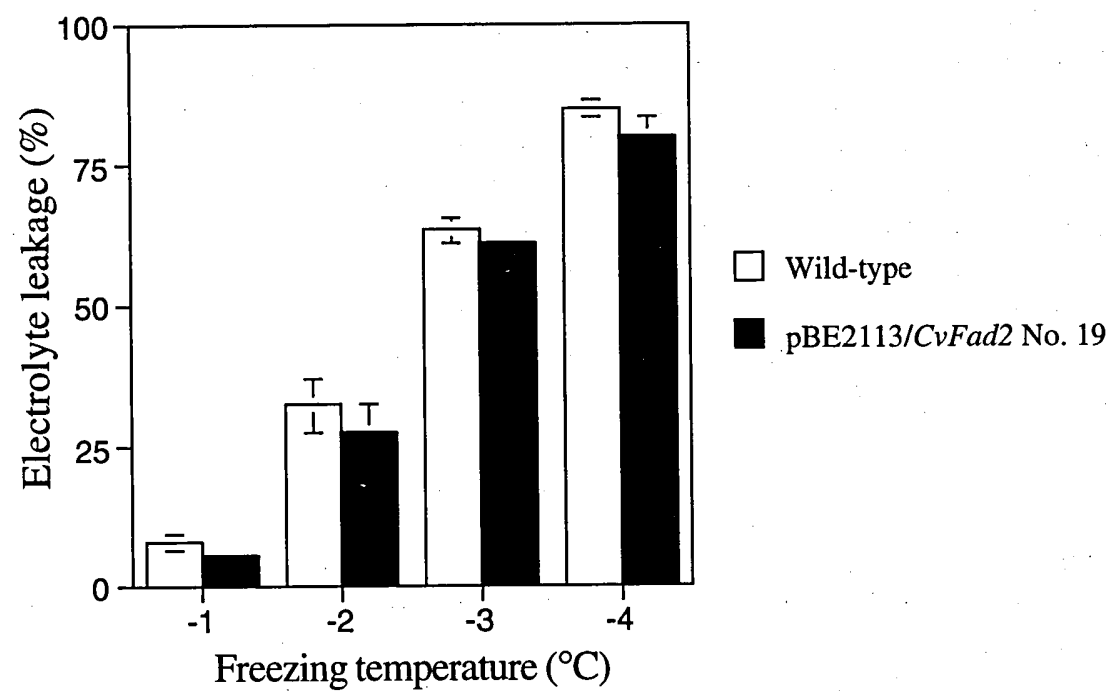

Fig. 5. Freezing tolerance of leaves of $C v F a d 2$ transgenic tobacco plant, assessed by measuring electrolyte leakage. The results shown are the average of at least three different experiments; three samples were used for each experiment. 


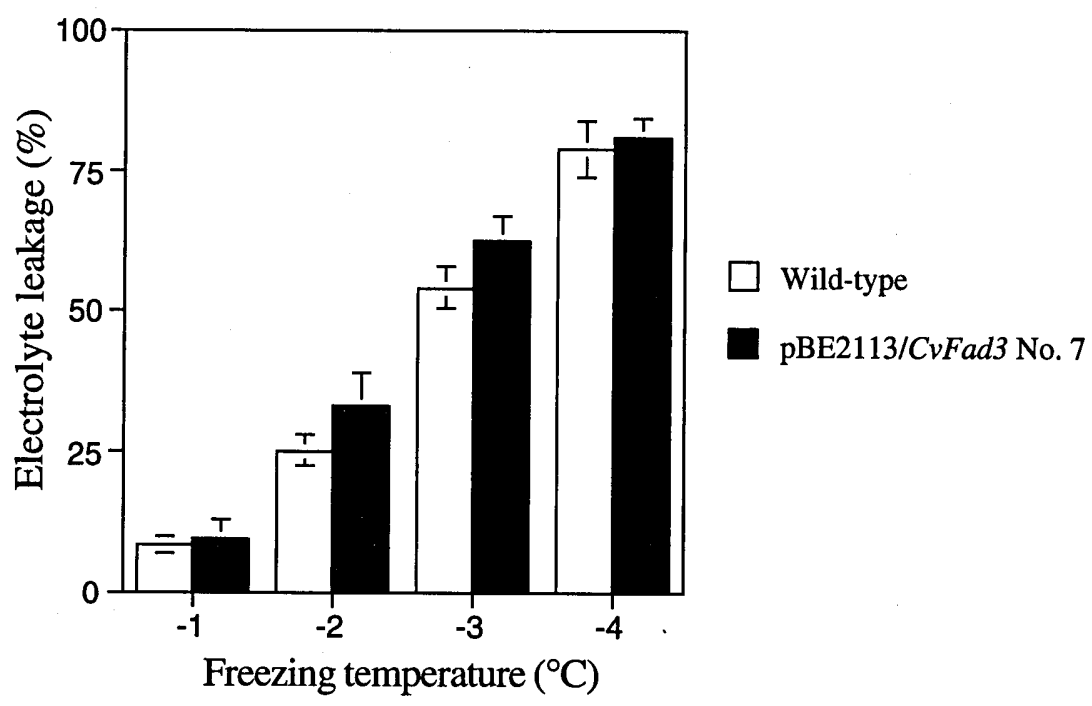

Fig. 6. Freezing tolerance of leaves of $\mathrm{CvFad} 3$ transgenic tobacco plant. For other details, see Fig. 5.

\section{Measurements of freezing tolerance of the leaves}

To assess freezing tolerance of the CvFad2 and CvFad3 transgenic tobacco plants, the electrolyte leakage (EL) of the leaves was measured after a freeze/thaw treatment, as described previously (Honjoh et al., 2001). Out of the CvFad2 transgenic lines, No. 19 line, which showed the highest unsaturation level, was selected as a representative of these plants. For the CvFad3 lines, No. 7 line was selected for the same reason.

The \%EL values of the CvFad2 plant were slightly lower than those of the wild-type plant at all temperatures investigated $\left(-1\right.$ to $-4^{\circ} \mathrm{C}$ ) (Fig. 5). Thus, the freezing tolerance of the CvFad2 plant was a little higher than that of the wild-type plant, although no significant differences in the \%EL were observed at all temperatures tested. Contrary to the case of $C v F a d 2$, the freezing tolerance of the $C v F a d 3$ plant tended to be slightly but not significantly lower than that of the wild-type at all the temperatures (Fig. 6).

\section{DISCUSSION}

For introduction of the CvFad2 gene into tobacco plants, we used a pBE2113 vector (Mitsuhara et al., 1996) with a strong promoter. Nevertheless, the levels of unsaturated fatty acids in the CvFad2 transgenic plants increased only slightly. According to the result of northern hybridization analysis, the CvFad2 gene was certainly expressed in the transgenic plants. The original, very low content of C18:1, the substrate for CvFAD2, in the wild-type may be a reason for the slight change in the fatty acid composition of the CvFad2 plants. 
In No. 7 line of $C v F a d 3$ transgenic plants, C18:3 content increased by $6.4 \%$ and $\mathrm{C} 18: 2$ decreased by $5.2 \%$ compared with wild-type. Different from C18:2, which is diminished by desaturation into C18:3, C18:3 seems not to be desaturated further (Ohlrogge and Browse, 1995). In addition, the content of $\mathrm{C} 18: 2$, the substrate for CvFAD3, was much higher than that of C18:1 originally in the tobacco plant. So the content of C18:3 might be easy to increase more than that of C18:2.

There was no correlation between the levels of CvFad genes transcription and those of fatty acid unsaturation. The No. 13 line of the CvFad3 transgenic plants, which showed the highest $C v F a d 3$ transcription level, did not increase the content of C18:3. So far the reason for this irreverence discrepancy remains to be elucidated.

A common hybridization band was detected in all plants including the wild-type and pBE2113-GUS plants by genomic Southern hybridization analysis using a CvFad3 probe. This band was probably derived from an endogenous fad3 gene. Because the hybridization was done in a relatively high stringent condition, the result suggests that the nucleic acid sequence of the CvFad3 is very similar to that of the tobacco endogenous fad3.

Measurement of the electrolyte leakage (EL) of the leaves showed that freezing tolerance of the CvFad2 plant was slightly higher than that of the wild-type plant. The EL method reflects the damage of cell membrane, so this result suggests that the increased unsaturated fatty acids content in cell membrane leads to the prevention of the plasma membrane from injury caused by freezing and thawing. Because the CvFad2 gene codes for microsomal FAD (Suga et al., 2002), increased unsaturated fatty acids would be derived from the plasma membrane.

The freeze-induced plasma membrane lesions can be classified mainly into two different forms dependent on freezing temperature (Uemura et al., 1995). Over the range from -2 to $-4^{\circ} \mathrm{C}$, the predominant membrane injury is "expansion-induced lysis", which is caused by the osmotic contraction and expansion cycle that occurs with freezing and thawing. At temperatures between -4 to $-8^{\circ} \mathrm{C}$, the predominant injury is freeze-induced lamellar to hexagonal II phase transitions, an interbilayer event that occurs when the plasma membrane is brought into close apposition with various endomembranes as a consequence of freeze-induced dehydration. In this study, a freezing tolerance test was done at -1 to $-4^{\circ} \mathrm{C}$, so the increased unsaturated fatty acids content seems to be a little protective against the expansion-induced lysis.

Freezing tolerance of the $\mathrm{CvFad} 3$ plant was slightly lower than that of the wild-type at all temperatures despite the unsaturation level of the plant being higher than that of the CvFad2 plant. In wheat, correlation of the acquisition of frost resistance and accumulation of linolenic acid was indicated (Willemot, 1977). Furthermore, when the plasma membrane of protoplasts isolated from nonacclimated rye leaves was artificially enriched with various $\mathrm{PC}$ species by adding them to the protoplasts in vitro, enrichment with 18:2/18:2-PC or 18:3/18:3-PC was the most effective in increasing the freezing tolerance, whereas fusion with saturated species had no effect (Steponkus et al., 1988). Therefore, desaturation of fatty acids in the plasma membrane is considered to be important for improving freezing tolerance. However, the freezing tolerance of $\mathrm{CvFad} 3$ plant did not increase but oppositely slightly decreased. Although it may not be applicable directly to the plant, in rat, an increase in the fatty acid double bond content in heart mitochondrial membranes leads to an increase in lipid peroxidation (Herrero et al., 2001). Because 
oxidative stress is one of the major stresses induced by chilling in chilling-sensitive plants (Hodges et al., 1997, Pinhero et al., 1997), unsaturated fatty acids might suffer too much oxidation, which leads to ion leakage from inside of the cell (Camp et al., 1996), during freezing tolerance tests. Simultaneous introduction of a gene encoding an enzyme, that protects plasma membrane lipids from peroxidation, such as GPx, G6PDH (Miki et al., 1996), SOD (Camp et al., 1996) or peroxidase (Yun et al., 2000) with CvFad3 may be effective in increasing freezing tolerance.

In this paper, we tried to investigate relationship between plasma membrane fatty acid desaturation and freezing tolerance. However, contribution of the desaturation to freezing tolerance has not yet been clarified. Freezing stress involves multiple stresses such as dehydration, low temperature and oxidation, and multiple genes are thought to be involved in freezing tolerance acquisition (Thomashow, 1998). So perhaps, it might be difficult to develop freezing tolerance of cold sensitive plants by introducing only a single gene. In other words, cooperation of multiple genes might be necessary to improve freezing tolerance. We hope that simultaneous introduction of gene(s), which concerns freezing tolerance, with CvFad gene(s) may help clarify the role(s) of the fatty acid desaturation.

\section{ACKNOWLEDGMENTS}

This research was supported by a Grant-in-Aid for Scientific Research (no. 13660127) from the Ministry of Education and Culture of Japan. The authors are grateful to Professor Norio Murata, National Institute of Basic Biology, Okazaki, Japan for kindly providing tobacco plants and Dr. Yuko Ohashi, National Institute of Agrobiological Resources, Tsukuba, Japan for a $\mathrm{pBE} 2113$ vector.

\section{REFERENCES}

Camp, W. V., K. Capiau, M. V. Montagu, D. Inzé and L. Slooten 1996 Enhancement of oxidative stress tolerance in transgenic tobacco plants overproducing Fe-superoxide dismutase in chloroplasts. Plant Physiol., 112: 1703-1714

Graham, D. and B. D. Patterson 1982 Responses of plants to low, non-freezing temperatures: proteins, metabolism and acclimation. Annu. Rev. Plant Physiol. Plant Mol. Biol., 33: 347-372

Guy, C. L. 1990 Cold acclimation and freezing tolerance: role of protein metabolism. Annu. Rev. Plant Physiol. Plant Mol. Biol., 41: 187-223

Hatano, S., H. Sadakane, M. Tutumi and T. Watanabe 1976 Studies on frost hardiness in Chlorella ellipsoidea I. Development of frost hardiness of Chlorella ellipsoidea in synchronous culture. Plant Cell Physiol., 17: 451-457

Herrero, A., M. Portero-Otin, M. J. Bellmunt, R. Pamplona and G. Barja 2001 Effect of the degree of fatty acid unsaturation of rat heart mitochondria on their rates of $\mathrm{H}_{2} \mathrm{O}_{2}$ Production and lipid and protein oxidative damage. Mech. Ageing Dev., 122: 427-443

Hodges, D. M., C. J. Andrews, D. A. Johnson and R. I. Hamilton 1997 Antioxidant Enzyme and compound responses to chilling stress and their combining abilities in differentially sensitive maize hybrids. Crop Sci., 37: 857-863

Honjoh, K., H. Shimizu, N. Nagaishi, H. Matsumoto, K. Suga, T. Miyamoto, M. Iio and S. Hatano 2001 Improvement of freezing tolerance in transgenic tobacco leaves by expressing the hiC6 gene. Biosci. Biotechnol. Biochem., 65(8): 1796-1804

Honjoh, K., M. Yoshimoto, T. Joh, T. Kajiwara, T. Miyamoto and S. Hatano 1995 Isolation and characterization of hardening-induced proteins in Chlorella vulgaris C-27: identification of late 
embryogenesis abundant proteins. Plant Cell Physiol., 36: 1421-1430

Jaglo-Ottosen, K. R., S. J. Gilmour, D. G. Zarka, O. Schabenberger and M. F. Thomashow 1998 Arabidopsis $C B F 1$ overexpression induces $C O R$ genes and enhances freezing tolerance. Science, 280: 104-106

Joh, T., T. Yoshida, M. Yoshimoto, T. Miyamoto and S. Hatano 1993 Composition and positional distribution of fatty acids in polar lipids from Chlorella ellipsoidea differing in chilling susceptibility and frost hardiness. Physiol. Plantarum, 89: 285-290

Kodama, H., G. Horiguchi, T. Nishiuchi, M. Nishimura and K. Iba 1995 Fatty acid desaturation during chilling acclimation is one of the factors involved in conferring low-temperature tolerance to young tobacco leaves. Plant Physiol., 107: 1177-1185

Miki, T., Y. Tsujimoto, S. Miyabe, K. Sugiyama, S. Izawa, Y. Inoue and A. Kimura 1996 Oxidative stress response in yeast: Purification and some properties of oxidative stress-inducible glucose-6-phosphate dehydrogenase from Hansenula mrakii. Biosci. Biotech. Biochem., 60(6): 966-970

Miquel, M., D. James, Jr., H. Dooner and J. Browse 1993 Arabidopsis requires polyunsaturated lipids for low-temperature survival. Proc. Natl. Acad. Sci. USA, 90: 6208-6012

Mitsuhara, I., M. Ugaki, H. Hirochika, M. Ohshima, T. Murakami, Y. Gotoh, Y. Katayose, S. Nakamura, R. Honkura, S. Nishimiya, K. Ueno, A. Mochizuki, H. Tanimoto, H. Tsugawa, Y. Otsuki and Y. Ohashi 1996 Efficient promoter cassettes for enhanced expression of foreign genes in dicotyledonous and monocotyledonous plants. Plant Cell Physiol., 37: 49-59

Ohlrogge, J. and J. Browse 1995 Lipid biosynthesis. Plant Cell, 7: 957-970

Pinhero, R. G., M. V. Rao, G Paliyath, D. P. Murr and R. A. Fletcher 1997 Changes in activities of antioxidant enzymes and their relationship to genetic and paclobutrazol-induced chilling tolerance of maize seedlings. Plant Physiol., 114: 695-704

Sadakane, H., K. Kabata and S. Hatano 1980 Studies on Frost Hardiness in Chlorella ellipsoidea.V. The role of glucose and related compounds. Environ. Exp. Bot., 20: 297-305

Seki, M., M. Narusaka, H. Abe, M. Kasuga, K. Yamaguchi-Shinozaki, P. Carninci, Y. Hayashizaki and K. Shinozaki 2001 Monitoring the expression pattern of 1300 Arabidopsis genes under drought and cold stresses by using a full-length cDNA microarray. The Plant Cell, 13: 61-72

Steponkus, P. L., M. Uemura, R. A. Balsamo, T. Arvinte and D. V. Lynch 1988 Transformation of the cryobehavior of rye protoplasts by modification of the plasma membrane lipid composition. Proc. Natl. Acad. Sci. USA, 85: 9026-9030

Suga, K., K. Honjoh, N. Furuya, H. Shimizu, K. Nishi, F. Shinohara, Y. Hirabaru, I. Maruyama, T. Miyamoto, S. Hatano and M. Io 2002 Two low-temperature-inducible Chlorella genes for $\Delta 12$ and $\omega-3$ Fatty Acid Desaturase (FAD): Isolation of $\Delta 12$ and $\omega-3$ fad cDNA Clones, Expression of $\Delta 12 \mathrm{fad}$ in Saccharomyces cerevisiae, and Expression of $\omega-3$ fad in Nicotiana tabacum. Biosci. Biotechnol. Biochem., 66(6): 1314-1327

Thomashow, M. F. 1998 Role of cold-responsive genes in plant freezing tolerance. Plant Physiol., 118: $1-7$

Uemura, M., R. A. Joseph and P. L. Steponkus 1995. Cold Acclimation of Arabidopsis thaliana. Plant Physiol., 109: 15-30

Wanner, L. A. and O. Junttila 1999 Cold induced freezing tolerance in Arabidopsis. Plant Physiol., 120: $391-399$

Webb, M. S., M. Uemura and P. L. Steponkus 1994 A comparison of freezing injury in oat and rye: Two cereals at the extremes of freezing tolerance. Plant Physiol., 104: 467-478

Willemot, C. 1977 Simultaneous inhibition of linolenic acid synthesis in winter wheat roots and frost hardening by BASF 13-338, a derivative of pyridazinone. Plant Physiol., 60: 1-4

Yun, B. W., G. H. Huh, H. S. Lee, S. Y. Kwon, J. K. Jo, J. S. Kim, K. Y. Cho and S. S. Kwak 2000 Differential resistance to methyl viologen in transgenic tobacco plants that express sweet potato peroxidases. J. Plant Physiol., 156: 504-509 